\title{
Research on Information Communication Channel and Monitoring of Sports Events
}

\author{
$\operatorname{Min} \mathbf{L i}^{1, \text { a }}$ \\ ${ }^{1}$ Jiangxi Science \& Technology Normal University, Jiangxi Province, Nanchang, 330013
}

Keywords: Sports Events; Communication Channels, Monitoring

\begin{abstract}
Due to the deepening of the process of social modernization, the interaction between people is becoming more and more closely, and the increase of the media makes the communication channel of information has been greatly expanded. As an important part of the information in the ocean of sports information, not only to enrich the spirit of the public life, but also allows sports events to a higher level of visibility. This paper first gives a brief overview of the definition of sporting events, and then analyzes the main communication channels of sports events, which are human communication, interpersonal communication, organization communication and mass communication. Finally, the research on the dissemination of sports events is systematically monitored method.
\end{abstract}

\section{Introduction}

As Chinese economic strength is growing, people's overall quality of life has been significantly improved, the degree of concern for the media is also growing and sport is the core content of the mass media an important part. Although the sporting event information for our daily life has brought convenience and happiness, but the proliferation of information will have a lot of consequences. It is necessary to straighten out the dissemination of information channels, with all kinds of effective way to monitor the information to expand the visibility of the event, so that positive and positive information can be healthy and rapid spread.

\section{The Main Dissemination Channels of Sports Information}

Spread Within the People. The so-called human spread, in fact, refers to the individual's minds, the main character of the information between me and my interaction, is the individual to obtain foreign information and in the human body to start the process of processing. This communication channel has the characteristics of concealment, thinking and short distance. And the spread of human beings will not be time and space constraints, can be carried out at any time, very free. Because the spread of people is mainly carried out in the heart, so subordinate to the field of psychology, due to the characteristics of concealment, resulting in the whole process of human transmission are difficult to in-depth investigation. But this does not mean that it will not happen, on the contrary, the spread of human beings is the basis of other kinds of communication channels. The dissemination of information on sports events refers to the end of the dissemination of information on the game process, event publicity and the overall quality of services such as self-evaluation, and to clarify their own attitude of the process. This communication channel will directly affect the audience whether to watch the game and memory sports news dissemination of the attitude, it must attach great importance.

Interpersonal Communication. Interpersonal communication is the most widespread spread of real life, but also the basic mass communication. Its core feature is the obvious feedback, the diversification of the media, and can effectively change the attitude, significant. The main thing in the sporting event is the interpersonal communication between the believers, the presence of interpersonal communication between the audience and the service workers, and the interpersonal communication among the athletes. With the rapid development of modern technology, interpersonal communication between the media is also evolving, people access to information 
channels are increasingly diversified, the total amount of trust has also been significantly improved. But the specific number is difficult to accurately calculate, so that the size of the huge intercourse between the spread of interpersonal communication is also difficult to investigate. After a sample survey of the audience found that the interpersonal communication occurred in the stadium on the efficient dissemination of sports information is particularly important, the scene for the organizing committee and the audience exchange and interaction has opened up an effective channel, the organizing committee must be fully through the interpersonal communication channels do the game publicity, try to make the audience feel satisfied with the game [1].

Organization Communication. The so-called organizational communication refers to the effective dissemination of the information dissemination activities carried out. Its core features are mainly the following points, the first communicator is the name of the group to speak, and secondly, most of the information disseminated are instructive and instructive color; finally it is carried out in an organized situation and formed a certain scale. Organizational communication of sporting event information, usually organized by the Organizing Committee held a reception, tournament conference and internal management meeting. Press conference held effectively, to enhance the transparency of information to protect the consumer's right to know, improve social supervision and adjust the image. In addition, the tournament conference can also be converted into a press conference and the main purpose is to convey the game to the audience of the relevant issues, the lifting of the concerns of consumers. The internal management meeting is usually in order to manage the internal staff, according to the progress of the game and information feedback, the assembly of different levels of staff meeting.

Mass Communication. The most prominent feature of mass communication is that the mode of communication is open and transparent, and there is no coercion, usually, the direction of transmission are one-way, feedback is generally after the end of the communication activities. Because the mass communication audience is very wide, so its influence and influence are very large, the transmission speed is also very fast, is the above three kinds of communication channels cannot be compared. As an indispensable part of social culture sports have a very important role in the evolution of national civilization. Sports events is the most exciting part of the entire sports culture, because of its both entertainment and ornamental, all kinds of international sports events have become the focus of the media scrambling to report. As the mass communication will have a direct impact on people's values, so naturally become sporting event information is one of the important channels of communication.

\section{The Monitoring of the Spread of Sporting Events Information}

The Monitoring of Information. The monitoring of information can be divided into two steps, the first is the analysis of information content, identify the authenticity of information. Followed by the discovery of effective information, it shows the objective facts and processes, to study these facts, the process of the objective situation and the first time to convey to the event organizing committee. In previous sports competitions, often appear gambling, taking doping and other bad events, sports events and sports organizations have brought a lot of negative effects. In the face of such problems, we must use the content communication method on the basis of dealing with loopholes in the operation mechanism of sports institutions. Facing the crisis caused by improper organization and management, we must face, take responsibility, and take effective measures to save.

The Monitoring of the Source. The monitoring of bad information is often a temporary solution and it must be effective monitoring of the source. For the Organizing Committee to effectively control the source, the organizing committee itself needs to take active measures. We must do a clear and reasonable division of labor, well-organized sports information on the dissemination of information on the release of information must be repeated review, improve the supervision mechanism, in the first time to grasp the direction of public opinion.

And the organizing committee is difficult to control the source mainly refers to the people watching the game and sports journalists. Although this kind of factors is difficult to control, but the 
organizing committee or to take active measures, before the game, carefully written to the various media, prepared a press conference, the characteristics of the event publicity, as to the audience Monitoring, it is mainly rely on public opinion [2].

The Monitoring of the Letter. Because information dissemination has the characteristics of complexity, it is difficult to monitor the new homes to each individual. Therefore, public opinion monitoring is the most critical means. Public opinion is a collection of attitudes, opinions and emotions expressed by people in various phenomena in real society. It has a relative persistence and consistency, and has a direct influence on the development of society. Therefore, it is of great practical significance to monitor it. The survey shows that if about $38.2 \%$ of people maintain a certain attitude, then this opinion in a certain range has a greater influence, if there are about $61.8 \%$ of people to maintain a certain attitude, then the view in a certain Within the scope of the dominant public opinion. In public opinion, rational and irrational elements are there, so the organizing committee must be a detailed analysis and judgment, and then do a good job in the management of the dredge.

The Monitoring of the Channel. The face of different sports events information dissemination channels, must use a different monitoring method. As the spread of people with a significant hidden, not easy to grasp the investigation, can do is to improve the information dissemination of the event environment and quality, resulting in an impact. For example, improve the attitude of the site service workers and service quality, to enhance the audience to watch the enthusiasm of the game and the athlete's degree of engagement, so that the game more brilliant. For the monitoring of interpersonal communication, it is mainly from the audience and on-site service personnel to carry out the spread between the start to carry out efficient monitoring. On the one hand, to strengthen the training of service workers, the organizing committee can set a number of outstanding service awards, after the end of the game to start selection, in the promotion of service personnel at the same time, so that the service level of the division to a higher level. Monitoring the communication between the audiences is usually done by the opinion leaders. Organizing Committee with the leaders of the division to establish contact, the first time the game information passed to them, and thus began to spread the second.

Organizational communication is the easiest to control, the organizing committee only need to control the content of good information, do a good job in the news conference on the line, but it should be noted that must not deliberately hide the negative information. For the mass communication, the Organizing Committee must cooperate with the major media to provide some feasible suggestions to the media, at the same time, make full use of the media channel to get the feedback, the content of the adjustment [3].

\section{Conclusion}

To sum up, there are four kinds of channels for information communication in sports events, namely, human communication, interpersonal communication, organization communication and mass communication. In order to avoid the indiscriminate proliferation of bad information, so the need for effective monitoring of the dissemination of sports information, I propose to information from the source, the source, the letter and the channel and other four different aspects to maintain the prosperity and stability of the sports information market.

\section{Acknowledgements}

Fund Project: Jiangxi Province Social Science "Thirteen" (2016) Planning Youth Project "Sports Event Information Dissemination and Monitoring", Project Number: $16 T Y 14$. 


\section{References}

[1] Fu Xiaoying, Zhang Desheng. Changes and Transformation of Crisis Communication Management in Sports Events in New Media Times - Taking Liaoning National Games as a Case [J]. Acta Metallurgica Sinica, 2015, 35 (3): 51-54.

[2] Zhang Yun. China's sports events news coverage of international communication influence research [J]. News knowledge, 2015, 12 (6): 102-103.

[3] Zhang Lu-ping.Model integration media sports events in the era of new exploration - TV media coverage of sports events innovation [J]. Sports, 2014, 15 (18): 134-134,113. 